\title{
Tradução e adaptação da Escala de Potencial de Ajustamento Intercultural para a realidade brasileira
}

\author{
Jesselyn Nayara Tashima - Universidade Federal de Uberlândia, Uberlândia/MG, Brasil \\ Sinésio Gomide Júnior - Universidade Federal de Uberlândia, Uberlândia/MG, Brasil
}

\begin{abstract}
Resumo
O presente estudo teve como objetivo adaptar a escala Intercultural Adjustment Potencial Scale (ICAPS) desenvolvida por Matsumoto e LeRoux (2006) para o contexto brasileiro. O processo de adaptação cultural seguiu cinco etapas: tradução do instrumento para o português, retrotradução do português para o inglês, avaliação semântica por comitê de juízes, préteste da versão de ICAPS em português e estudo das características psicométricas da escala. Os dados foram submetidos ao cálculo da confiabilidade dos fatores originais de ICAPS e às análises fatoriais necessárias à adaptação e validação do instrumento. Os resultados apontaram para uma estrutura que não apresentou suporte empírico para o contexto brasileiro. Discutiram-se as limitações teóricas do construto em estudo e possíveis equívocos na construção dos itens do instrumento.
\end{abstract}

Palavras-chave: Migração humana; Ajustamento; Psicometria.

Translation and adaptation of the Intercultural Potential Adjustment Scale to the brazilian context

\begin{abstract}
This study aimed to adapt the Intercultural Adjustment Potential Scale (ICAPS), developed by Matsumoto and LeRoux (2006) to the Brazilian context. The adaptation process followed five steps: translation to Portuguese, back translation to English, semantic analysis by expert committee and pretest of the ICAPS version in Portuguese and study of the psychometrics properties of the scale. The data was submitted to reliability calculus of the original factors of ICAPS and to factorial analysis needed for the adaptation and validation of the instrument. The results pointed out a structure that didn't present empirical support for the Brazilian context. It has discussed the theoretical limitations to the construct and possible mistakes on the construction of the items of the instrument.

Keywords: Psychological instrument; Cultural adaptation; Psychometrics.
\end{abstract}

Traducción y adaptación de la Escala de Potencial de Adaptación Intercultural para el contexto brasileño

\begin{abstract}
Resumen
El presente estudio tuvo como objetivo adaptar la escala Intercultural Adjustment Potencial Scale (ICAPS) desarrollada por Matsumoto y LeRoux (2006) para el contexto brasileño. El proceso de adaptación cultural siguió cinco etapas: traducción del instrumento para el portugués, retrotraducción del portugués para el inglés, evaluación semántica por el comité de jueces, pre-test de la versión de ICAPS en portugués y estudio de las características psicométricas de la escala. Los datos fueron sometidos al cálculo de la confiabilidad de los factores originales de ICAPS y a los análisis factoriales necesarios para la adaptación y validación del instrumento. Los resultados indicaron una estructura que no presentó soporte empírico para el contexto brasileño. Se discutieron las limitaciones teóricas de la construcción en estudio y posibles equívocos en la misma de los ítems del instrumento.

Palabras clave: Emigración humana; Ajustamiento; Psicometría.
\end{abstract}

Ajustamento intercultural é definido como um processo motivacional em que o migrante objetiva modificar seu comportamento e crenças a fim de minimizar os efeitos negativos da migração e potencializar os efeitos positivos (Sussman, 2000). Outros autores definem este processo como uma experiência subjetiva de bem-estar (Matsumoto, Hirayama \& LeRoux, 2005), aceitação, satisfação (Brislin, 1981) e conforto psicológico experimentado pelos indivíduos acerca dos vários aspectos do novo ambiente (Black, 1990; Nicholson, 1984; Oberg, 1960). De forma complementar, Matsumoto e cols. (2007) trazem, ainda, que o ajustamento intercultural inclui autoconhecimento, autoestima, estado de humor e saúde.

O ajustamento intercultural também pode ser entendido como um fenômeno que envolve aspectos objetivos e subjetivos da migração. Os aspectos subjetivos incluem as emoções como raiva, tristeza, Disponivel em www.scielo.br satisfação, enquanto os fatores objetivos do ajustamento intercultural compreendem aspectos externos aos indivíduos, como, por exemplo, condições de trabalho e moradia (Yoo, Matsumoto \& Le Roux, 2006).

Ward (1996) diferencia o ajustamento em psicológico e sociocultural. O ajustamento psicológico refere-se ao estado emocional, afetivo, à satisfação geral do indivíduo e ao bem-estar provocado pela experiência intercultural. É influenciado por características de personalidade, mudanças de vida, suporte social e estilo de enfrentamento pela qualidade do contato que o indivíduo estabelece com a população local. Já o ajustamento sociocultural está diretamente relacionado à habilidade em lidar com problemas do cotidiano familiar e social (Ward \& Searle, 1991; Ward \& Kennedy, 1993). Em um nível cognitivo, o ajustamento sociocultural é a maneira como o indivíduo percebe a nova cultura; em termos 
comportamentais é como ele estabelece padrões de comunicação e interação com os anfitriões e, em nível psicológico, é a confiança e conforto percebidos diante do novo ambiente. Esse tipo de ajustamento é influenciado por fatores como conhecimento cultural, tempo de estadia, fluência no idioma e distância cultural. Nesse caso, a quantidade de interação é indicador de ajustamento sociocultural (Ward \& Searle, 1991; Ward \& Kennedy, 1993).

$\mathrm{Na}$ literatura sobre ajustamento intercultural há a predominância de três abordagens mais amplamente divulgadas na área (Shupe, 2007). Uma das vertentes examina as consequências de viver em uma cultura distinta, considerando o ajustamento como uma variável independente e preditiva. Essa abordagem busca trabalhar com as mudanças de vida, frequentemente negativas, que acompanham $\mathrm{O}$ processo de migração. Temas como estresse e enfrentamento são bastante comuns nos trabalhos divulgados por essa corrente.

Uma segunda abordagem considera o ajustamento como uma variável dependente ou variável critério e busca avaliar os fatores que podem predizer um ajustamento bem sucedido. Para os defensores dessa abordagem, existe uma série de variáveis que predizem ajustamento intercultural: suporte social e autoeficácia (Tsang, 2001), comunicação competente (Zimmerman, 1995), interação com os anfitriões (Tsang, 2001) e fatores de personalidade (Shupe, 2007).

Já um terceiro grupo de pesquisadores tem desenvolvido estudos que descrevem a dinâmica do ajustamento intercultural. Baseados em uma abordagem de aprendizagem, esses estudiosos consideram a aquisição de habilidades e conhecimentos como fator precursor de um melhor ajustamento. A partir dessa perspectiva, os teóricos dessa abordagem descrevem, com base em curvas de ajustamento, as fases do processo de interação intercultural (Shupe, 2007).

Grande parte desses modelos teóricos serviu como referencial para a construção de instrumentos de avaliação do ajustamento intercultural. Inicialmente, muitos instrumentos surgiram para explicar o fracasso de profissionais no exterior, predizer sucesso no exterior, desenvolver estratégias de seleção e programar e testar treinamentos com estrangeiros. Essas ferramentas tinham como população-alvo empresários expatriados. Mais tarde, por causa da globalização e da grande repercussão do tema, foram criados instrumentos com objetivos e com populações bem variados (Ruben, 1989).

Além dos objetivos diversos, os instrumentos também diferem quanto aos construtos a serem avaliados tomando por base a temática do ajustamento.
Enquanto alguns instrumentos têm sido designados para medir os preditores ou antecedentes do ajustamento intercultural (como, por exemplo, os traços de personalidade), outras ferramentas têm investigado fatores como habilidades no idioma, familiaridade com a cultura anfitriã e aspectos demográficos (Matsumoto \& cols., 2001). Um desses instrumentos criados foi o Intercultural Adjustment Potencial Scale (ICAPS) construído por Mastumoto e LeRoux (2006) com o objetivo de mensurar o grau de dificuldade de uma pessoa ao se ajustar a uma nova cultura (Matsumoto \& LeRoux, 2006).

Baseado nos achados da literatura sobre estresse e enfrentamento, Matsumoto e cols. (2003) optaram por avaliar as variáveis preditoras do ajustamento psicológico e defendem a ideia de que algumas habilidades psicológicas, como flexibilidade, regulação emocional, pensamento crítico ou criatividade e abertura a novas experiências, são determinantes ao se pensar o ajustamento intercultural. Assim, esses autores (Matsumoto \& cols., 2003) acreditam que o processo de migração a uma nova cultura é um evento que abrange situações estressantes que requerem um conjunto de habilidades psicológicas para enfrentar os novos desafios impostos pela cultura anfitriã. Logo, o desenvolvimento ou melhoria dessas habilidades se faz necessário a fim de minimizar o efeito do conflito e do estresse, muitas vezes inevitáveis no processo de ajustamento intercultural. Tais habilidades são primordiais para que o indivíduo se engaje na assimilação de novos esquemas de interação cultural, comportamental e cognitiva. Como efeito dessa expansão de repertório de respostas, formas positivas e construtivas de lidar com o novo ambiente podem ser desenvolvidas (Matsumoto \& cols., 2001).

Os mesmos pesquisadores deram início à criação da escala com a realização de grupos focais estruturados com japoneses residentes nos Estados Unidos, tendo a finalidade de identificar os principais conflitos e problemas interculturais enfrentados por esse grupo em seu cotidiano (Matsumoto \& cols., 2001). Além disso, os autores de ICAPS também partiram de análises de vários inventários de personalidade com o intuito de extrair construtos psicológicos relacionados ao tema de adaptação intercultural que eles julgaram ser relevantes, como: regulação emocional, pensamento crítico, abertura, flexibilidade, segurança interpessoal, comprometimento emocional com a maneira tradicional de pensar, tolerância à ambiguidade e empatia. Os instrumentos analisados foram: o Eysenck Personality Inventory (EPI), o Beck Depression Inventory (BDI), State-Trait Anxiety Inventory (STAI), Bem Sex Role Inventory (BSRI), o original Minnesota Multiphasic Personality Inventory 
(MMPI), o California Personality Inventory (CPI), o NEO Personality Inventory (NEO-PI), o Big 5 Personality Inventory (BFI), o California F-Scale e o Interpersonal Reactivity Index (Matsumoto \& cols., 2001).

Com as informações obtidas nesses dois momentos, foram elaborados 193 itens que buscaram abranger habilidades presumidas como necessárias aos indivíduos para lidar com o conflito e estresse do processo de inserção em um novo contexto cultural. Foi utilizado o coeficiente de correlação de Pearson para excluir os itens que apresentassem pouca correlação entre si. Assim, 40 itens foram excluídos nessa fase (Matsumoto et al., 2001).

Em um segundo momento, os autores buscaram correlacionar os 153 itens resultantes do primeiro estudo com a proficiência no idioma (inglês), problemas específicos encontrados na adaptação ao novo contexto e das dificuldades individuais observadas nos grupos focais em ajustar à vida nos Estados Unidos. Essa fase levou à redução para 55 itens, em que foram descartados os com baixa correlação com o critério empírico (Matsumoto \& cols., 2005).

Em seguida, foi realizada a análise fatorial do instrumento, que sugeriu a presença de quatro fatores, que os autores (Matsumoto \& cols., 2001) nomearam de regulação emocional (habilidade de modular as reações emocionais a fim de evitar defesas psicológicas), abertura (habilidade de se engajar no aprendizado de uma nova cultura), flexibilidade (ser livre de excessivas ligações com a maneira anterior de pensar e estar disposto a tolerar a ambiguidade) e pensamento crítico ou criatividade (habilidade de gerar hipóteses novas e criativas sobre incidentes da nova cultura que vão além dos sistemas da cultura original) (Matsumoto \& cols., 2001). Ainda de acordo com os autores do instrumento, depois de realizada a análise fatorial da escala, verificou-se que somente 26 itens foram capturados pelos quatro fatores. Assim, neste estudo, optou-se por trabalhar com apenas os 26 itens capturados pelos quatro fatores originais da escala.

Os autores avaliaram a confiabilidade do instrumento a partir do cálculo da consistência interna e do teste-reteste. Argumentam os mesmos autores ser o teste-reteste uma técnica mais adequada para avaliar a confiabilidade do instrumento, já que este foi construído por meio de métodos empíricos e por comportar mais de um fator. Dessa maneira, apesar de apresentar apenas uma moderada consistência interna $(\alpha=0,78$ e $\alpha=0,51$, nos primeiros estudos), a confiabilidade teste-reteste tem sido alta na maior parte das amostras pesquisadas $(r>0,76)$ (Matsumoto \& LeRoux, 2006).
Os autores conduziram 17 estudos em que puderam documentar e demonstrar a fidedignidade e validade da escala ao predizer o ajustamento intercultural. As evidências apontam para um instrumento que foca em habilidades psicológicas teórica e empiricamente consideradas importantes ao lidar com o conflito e o estresse intercultural (Matsumoto \& cols., 2001). Além disso, de acordo com Matsumoto e cols. (2001), ICAPS é um dos poucos instrumentos existentes atualmente que pode ser utilizado em uma variedade de populações, incluindo estudantes, empresários, donas de casa e indivíduos envolvidos em casamentos interculturais.

Outros estudos realizados por pesquisadores da Áustria também comprovaram a eficácia de ICAPS ao predizer ajustamento intercultural (Savick, DowningBurnette, Hellen, Binden \& Suntingen, 2004). Os mesmos pesquisadores conduziram uma pesquisa com os objetivos de comparar os escores obtidos em ICAPS com escores em ajustamento em duas populações uma de estudantes de intercâmbio e uma outra amostra com estudantes que permaneceram no país de origem; examinar os escores de ICAPS dos estudantes de intercâmbio em cinco momentos da jornada de intercâmbio um mês antes do embarque, no início, no meio e no final da temporada, e três meses depois do intercâmbio; avaliar se ICAPS e escores de ajustamento individual dos estudantes de intercâmbio aumentam ao longo da estadia na cultura estrangeira; e, por último, avaliar se ICAPS e ajustamento pessoal estão relacionados a características de personalidade e estratégias de enfrentamento. Os resultados encontrados sugeriram que os participantes da pesquisa que tiveram maiores escores em ICAPS também apresentaram altos níveis de extroversão, abertura para novas experiências, e se mostraram mais positivos e esperançosos em suas próprias habilidades. Esses indivíduos ainda apresentaram estratégias específicas ao lidar com estresse e ansiedade no novo ambiente (Savick \& cols., 2004).

Diante do exposto, observa-se que o processo de ajustamento a uma nova cultura é um fenômeno complexo e abrange aspectos sociais, culturais e psicológicos. Há, contudo, que se considerar a escassez de pesquisas realizadas no Brasil com o tema de ajustamento intercultural de migrantes e a inexistência de instrumentos específicos para avaliar o potencial de ajustamento intercultural que seja construído ou adaptado à população brasileira.

No entanto, faz-se necessário adaptar culturalmente um instrumento já validado e disponível em outro idioma e utilizado por outros pesquisadores ou construir um novo instrumento para avaliar o construto estudado. Assim, nesta pesquisa buscou-se 
adaptar a Escala de Potencial de Ajustamento Intercultural (ICAPS) ao contexto brasileiro, testar e verificar suas características psicométricas. Para a adaptação da escala para o Brasil, procurou-se seguir um protocolo de procedimentos de adaptação transcultural de instrumentos de medida com a finalidade de garantir uma melhor compreensão possível para a população-alvo, bem como proporcionar uma equivalência entre o instrumento original e a versão brasileira.

\section{Método}

\section{Participantes}

A amostra foi composta por 428 estudantes da cidade de Uberlândia, sendo 17 do Ensino Fundamental, 123 do Ensino Médio e 285 do Ensino Superior, com 3 casos omissos. A faixa etária variou de 14 a 46 anos, com média de 19,21 anos e desvio padrão de 3,97, sendo 223 nascidos em Uberlândia e 186 nascidos em outras cidades.

\section{Instrumento}

A Intercultural Adjustment Potencial Scale (ICAPS) foi desenvolvida por Matsumoto e cols. (2001) com o objetivo de mensurar de maneira válida e confiável o grau de dificuldade de uma pessoa ao se ajustar em uma nova cultura (Matsumoto \& LeRoux, 2006). Em seus trabalhos, esses autores optaram por focar nos aspectos referentes à psicologia social do ajustamento intercultural (Matsumoto \& cols., 2001). Essa equipe de pesquisadores partiu da literatura sobre estresse e enfrentamento para desenvolver a escala ICAPS. Essa teoria, além de enfatizar a importância dos grandes eventos de vida na saúde mental dos indivíduos, também considera que os eventos cotidianos acarretam consequências positivas e negativas para o bem-estar e os relacionamentos dos indivíduos. Essa concepção se torna muito mais evidente ao se tratar de encontros interculturais em que o cotidiano dos imigrantes é repleto de conflitos e ambiguidades.

Além disso, assim como na literatura de estresse e enfrentamento, a emoção desempenha um papel primordial no ajustamento intercultural. Os mesmos autores recorrem a Tomkins (1963) ao utilizar a definição de emoção em seus trabalhos. Para ele, a emoção é a base de motivação para qualquer comportamento; sendo assim, para compreender o comportamento de uma determinada pessoa, faz-se necessário entender as suas emoções (Tomkins, 1963). Sob essa perspectiva, Matsumoto e cols. (2001) buscaram adotar um modelo de ajustamento intercultural em que o controle das emoções e outras habilidades psicológicas (pensamento crítico, flexibilidade e abertura) desempenham papéis determinantes para um ajustamento intercultural bem sucedido.

A análise fatorial do instrumento sugeriu a presença de quatro fatores, que os autores (Matsumoto \& cols., 2001) nomearam: regulação emocional (Emotional Regulation) - habilidade de monitorar e manejar as expressões e experiências emocionais e engajar em pensamentos claros sobre os incidentes interculturais sem recorrer a defesas psicológicas; abertura à experiência (Openness to Experience) - abertura a novas experiências, emoções e pensamento; flexibilidade (Flexibility) - habilidade de assimilar novas experiências, esquemas e novas formas de pensar, ser livre de excessivas ligações com a maneira anterior de pensar e estar disposto a tolerar a ambiguidade; e pensamento crítico (Critical Thinking) - habilidade de gerar hipóteses novas e criativas sobre incidentes da nova cultura que vão além dos sistemas da cultura original.

Os resultados encontrados na análise fatorial do instrumento sugerem que há itens individuais que não foram capturados pelos 4 fatores, já que dos 55 itens resultantes desta última análise, somente 26 itens foram capturados pelos fatores. Assim, neste estudo, optou-se por trabalhar com apenas os itens capturados pelos 4 fatores, o que totaliza 26 itens (Matsumoto \& LeRoux, 2006). Em cada uma das situações (itens) do questionário, o participante deverá escolher uma entre as sete respostas possíveis, dadas em uma escala tipo likert na qual 7 (sete) corresponde a concordo totalmente e 0 (zero) a discordo totalmente.

\section{Procedimentos}

Neste estudo, o processo de equivalência transcultural foi baseado no roteiro de Beaton, Bombardier, Guillemin e Ferraz (2000) com modificações, o qual prevê algumas etapas metodológicas, sendo elas: a tradução, a retrotradução, a revisão por um comitê de especialistas, o pré-teste e a verificação das características psicométricas.

Para a tradução e retrotradução, foi enviado um convite por e-mail aos tradutores para participarem da pesquisa. Após a confirmação de ambos, o instrumento foi enviado por e-mail primeiramente para a tradutora, e em seguida, o instrumento traduzido para $\mathrm{O}$ português foi enviado ao retrotradutor para ser realizada a tradução reversa para o inglês. Ao final de cada tradução, os instrumentos eram enviados aos autores deste estudo para apreciações e revisão da versão em português. Assim, a versão retrotraduzida era comparada item por item com a versão original em inglês tendo em vista a equivalência semântica de cada termo do instrumento. 
Para a realização da análise semântica do instrumento, o critério escolhido para inclusão dos participantes nessa etapa era de que os sujeitos tivessem pelo menos ensino superior completo. Sendo assim, a amostra foi composta por treze sujeitos (juízes). Quanto ao gênero, oito juízes eram do gênero feminino e cinco do gênero masculino. Em relação ao grau de instrução, três são doutores em psicologia, sendo dois em Psicologia Social e do Trabalho, dois mestres em psicologia e oito com curso superior em áreas do Direito, Relações Públicas, Psicologia e Administração. Para essa etapa, foi utilizado um questionário contendo perguntas referentes aos dados complementares, como gênero e grau de instrução dos juízes, as instruções iniciais e os 26 itens da escala ICAPS traduzidos para o português com o espaço de uma linha abaixo de cada frase para possíveis sugestões e alterações da redação, tendo em vista as palavras de difícil compreensão, sentido dúbio ou incompleto. Esse procedimento teve como objetivo analisar os itens do ponto de vista semântico, para que a linguagem fosse considerada de fácil compreensão por estudantes brasileiros.

Após essa etapa, a versão traduzida para o português foi apresentada a uma amostra da população alvo, composta por 49 estudantes do curso de psicologia de uma universidade federal do Triângulo Mineiro e teve como objetivo verificar o entendimento quanto às instruções e frases do instrumento. Inicialmente, foi explicado o objetivo da pesquisa e a forma de participação, e, em seguida, o instrumento foi reproduzido em voz alta, item por item, perante todo o grupo, e os sujeitos foram questionados sobre a existência de termos de difícil compreensão ou sentido dúbio.

O projeto foi aprovado pelo Comitê de Ética (CEP-UFU) sob protocolo de número 350-10. Inicialmente, foi feito um contato com os coordenadores de alguns cursos de uma universidade federal do Triângulo Mineiro e de uma Instituição de Cursos Profissionalizantes para que, com o consentimento destes, pudesse ser realizada a etapa de coleta de dados nas salas de aulas.

Ao se dirigir às salas de aula, a aplicadora solicitava a autorização do professor, explicava-lhe os objetivos do trabalho e pedia sua autorização para falar com os alunos. A eles, explicava os objetivos do estudo, ressaltava o anonimato de suas respostas, esclarecendo que os questionários não eram identificados, e solicitava autorização formal para colaborar, pedindo-lhes que assinassem um termo de consentimento no qual constava o compromisso de sigilo por parte dos pesquisadores e a garantia de que nenhum prejuízo lhes adviria de suas respostas. Foi- lhes explicado que as conclusões do estudo resultariam das respostas do grupo e que nenhum questionário seria analisado individualmente. Após consentimento dos alunos, estes foram convidados a responder de acordo com o que estava sendo pedido. Foi explicado, também, que qualquer dúvida com relação ao correto preenchimento da escala poderia ser discutida a qualquer momento e, caso algum participante decidisse abandonar a participação na pesquisa durante a aplicação do instrumento, poderia fazê-lo, sem prejuízo algum. Após preenchimento dos alunos, a escala era recolhida e lacrada em envelopes sem a identificação dos participantes.

\section{Resultados}

Para a tradução, a tradutora foi informada de que se tratava de uma adaptação transcultural de um instrumento de medida psicológica, sendo imprescindível que se buscasse preservar o mesmo significado ou sentido dos itens, a fim de manter a integridade do instrumento. A retrotradução foi realizada para se comparar a versão do instrumento retrotraduzido com a versão original em inglês. Quanto à avaliação da equivalência semântica entre a versão retrotraduzida e a versão original, realizada pelos autores do estudo, observou-se que a maior parte dos itens (24) obteve entre $91,66 \%$ e $100,0 \%$ de concordância semântica. Apenas 2 itens apresentaram concordância de $66,67 \%$ e $71,42 \%$, respectivamente. $\mathrm{Na}$ análise do significado geral, a maioria dos itens foi considerada de significado inalterado em relação ao instrumento original. Nenhum item foi avaliado como completamente alterado. Entre os itens de menor concordância, não foi detectado nenhum erro ou discrepância grave, que poderia prejudicar o sentido dos itens em relação ao instrumento original. Esse processo resultou na versão traduzida do instrumento.

A análise semântica do instrumento consistiu na submissão da escala original a treze juízes, com o objetivo de avaliarem a compreensão dos itens da escala. Foi solicitado que colaborassem oferecendo sugestões ao conteúdo, com expressões e frases, tornando-as mais compreensíveis ao contexto brasileiro. Algumas modificações foram propostas pelos juízes quanto à semântica do instrumento, e três juízes não fizeram nenhuma alteração. Contudo, verificou-se que não houve nenhuma concordância entre as alterações propostas pelos juízes. Sendo assim, optou-se por manter os itens traduzidos sem nenhuma alteração.

$\mathrm{Na}$ fase do pré-teste, não houve nenhum questionamento significativo, o que indicava que o instrumento apresentava-se de maneira compreensível 
à população participante. Novamente, optou-se por manter a versão traduzida para o português sem nenhuma alteração.

Os dados foram analisados utilizando-se $\mathrm{O}$ programa estatístico SPSS (Statistical Package of Social Science, versão 15.0). As análises descritivas e exploratórias investigaram a exatidão da entrada dos dados, a distribuição dos casos omissos, o tamanho da amostra, os casos extremos. Essas análises não apontaram vieses que inviabilizariam o estudo. Os casos omissos e os extremos não excederam a porcentagem de $5 \%$ em cada uma das variáveis (itens) do instrumento (Tabachnick \& Fidell, 2007) e o número de respondentes mostrou-se adequado quando comparado ao número de itens. Pasquali (2005; 2010) recomenda uma proporção de até 10 respondentes por item. Neste estudo, a proporção é de 16,46 respondentes por item.

As quatro dimensões originais de ICAPS (Intercultural Adjustment Potencial Scale) foram submetidas ao cálculo do coeficiente de alfa de Cronbach, com o objetivo de verificar a confiabilidade do instrumento na amostra da pesquisa. Para o fator Regulação Emocional o valor encontrado foi de -0,029; para o fator abertura, o índice foi de 0,087 ; no fator flexibilidade o $\alpha$ foi de 0,085; para o fator pensamento crítico, o valor foi de 0,228. Assim, de acordo com a literatura (Pasquali, 2005), os resultados indicaram uma fidedignidade insatisfatória para todos os fatores da escala (ver Tabela 1).

Tabela 1. Resumo das características dos itens originais de ICAPS e indice de confiabilidade da amostra de adaptação de validação

\begin{tabular}{lccc}
\multicolumn{1}{c}{ Instrumento } & Fatores & Número dos itens & $\begin{array}{c}\text { Alfa de Cronbach } \\
\text { (amostra) }\end{array}$ \\
\hline & Regulação emocional & $2,5,6,7,9,10,15$, & $-0,029$ \\
& Abertura & $3,12,18$ & 0,087 \\
$\begin{array}{l}\text { Escala de Potencial de } \\
\text { Adaptação Intercultural }\end{array}$ & Flexibilidade & $1,8,19,20,25,26$ & 0,085 \\
& Pensamento crítico & $4,11,16,20,21,22,23$ & 0,228 \\
\hline
\end{tabular}

O atendimento das condições necessárias para a análise fatorial foi verificado a partir do índice de adequação da amostra, o Kaiser-Meyer-Olkin (KMO), que teve valor de 0,63, caracterizado como satisfatório (Aaker, Kumar \& Day, 2001). O KMO é uma estatística que indica a proporção de variância dos dados que pode ser atribuída a uma variância comum a todas as variáveis. Valores próximos de 1,0 são desejáveis e acima de 0,80 são considerados satisfatórios. O teste de esfericidade de Bartlett obtido foi de $x 2=1196,36 ; \mathrm{p}<0,01$, o que demonstra a rejeição da hipótese nula, assumida como a falta de correlação entre os itens, identificada pela matriz identidade (Hair \& cols., 2006). Assim, essas estatísticas demonstram que, embora haja correlações entre os itens (que poderia caracterizá-los como fatores, já que agrupáveis), demonstram, igualmente, a possibilidade de uma dispersão de agrupamentos, o que, em princípio, dificultaria sua interpretabilidade quando confrontados com o instrumento original constituído por apenas quatro fatores.

Para estimar o número de componentes, foi realizada uma análise exploratória dos dados por meio da Análise dos Componentes Principais - ACP. O resultado obtido indicou uma estrutura de 10 componentes com autovalores acima de 1 , porém o scree plot indicou uma mudança na direção da curva no quarto fator, sugerindo uma solução com três ou quatro fatores, conforme Figura 1. 


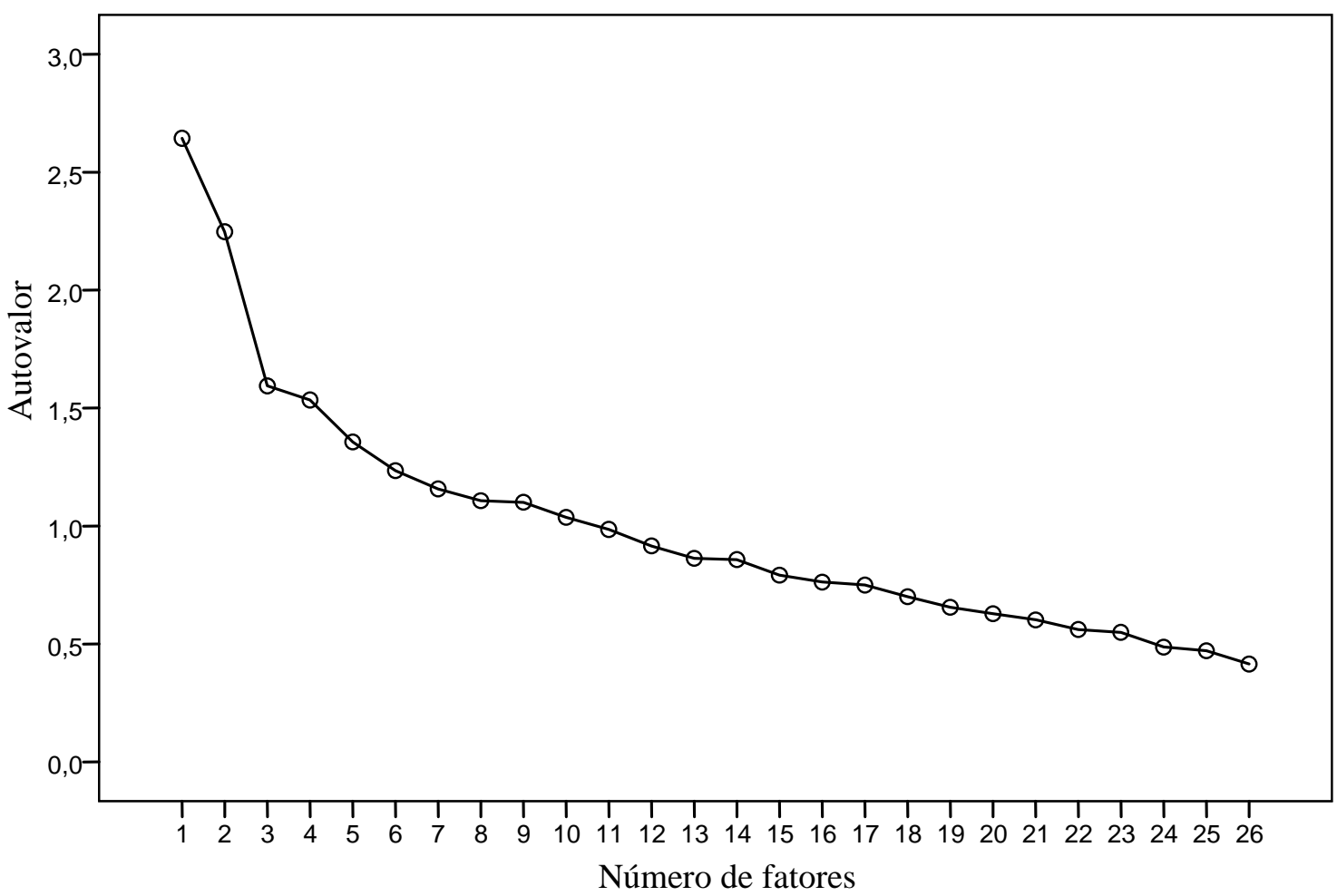

Figura 1. Scree plot dos itens da ICAPS.

Os dados foram posteriormente submetidos à fatoração dos eixos principais (Principal Axis Factoring PAF). A partir dos resultados encontrados anteriormente no cálculo do PC, adotaram-se como critérios uma estrutura fatorial composta por quatro fatores e itens com cargas fatoriais iguais ou superiores a 0,30 . O próximo passo foi escolher o tipo de rotação dos fatores. Tendo em vista a pressuposição da correlação (dependência) entre os fatores, (Pasquali, 2005; Tabachinick \& Fidell, 2007), o método rotacional escolhido foi o oblíquo (Direct Oblimin).

Os resultados apontaram para uma solução composta pelos quatro fatores, que explicaram 30,78\% da variância total do construto, sendo que o fator 1 foi responsável pela maior explicação da variância com $10,07 \%$, seguido pelo fator 2 , com $8,68 \%$ de explicação, pelo fator 3 , com $6,10 \%$ e pelo fator 4 , com
$5,91 \%$ (Tabela 2). Os autovalores foram maiores que 1,5 nos quatro fatores, e as cargas fatoriais dos itens em seus respectivos fatores foram superiores a 0,30 .

O valor do alfa de Cronbach para o fator 1 foi de 0,10; para o fator 2, de $-0,35$; para o fator 3, de -0,04; e para o fator 4, de 0,45 (ver Tabela 2). Conforme Pasquali $(2005 ; 2010)$, os valores de confiabilidade considerados adequados são aqueles superiores a $0,70-$ para pesquisas acadêmicas - ou superiores a 0,80 , para a realização de diagnósticos. Acrescente-se a esses resultados a variância explicada por cada um dos fatores obtidos. Parece haver aí uma indicação de que o instrumento resultante das análises não encontra correspondência ao constructo que se pretendeu mensurar. As implicações desses resultados são apresentadas a seguir. 
Tabela 2. Composição dos fatores, variâncias explicadas, valores próprios, cargas fatoriais dos itens e alfa de Cronbach dos fatores

\begin{tabular}{|c|c|c|c|c|c|}
\hline Itens & F1 & F2 & F3 & F4 & Comunalidades \\
\hline Assistir a espetáculos de balé, ou dança moderna, é chato. & $-0,42$ & & & & 0,30 \\
\hline Eu gosto de poemas tradicionais. & 0,54 & & & & 0,30 \\
\hline Eu já tentei escrever poesia. & 0,58 & & & & 0,34 \\
\hline Eu gosto de pensar sobre as origens do universo & 0,34 & & & & 0,12 \\
\hline Estar em situações emocionais tensas me assusta. & & 0,68 & & & 0,48 \\
\hline Eu não me preocupo muito. & & $-0,38$ & & & 0,16 \\
\hline Eu raramente me sinto ansioso ou com medo. & & $-0,51$ & & & 0,28 \\
\hline Eu não sinto muito prazer em conversar com as pessoas. & & & 0,38 & & 0,16 \\
\hline Eu geralmente me sinto inferior aos outros. & & & 0,30 & & 0,19 \\
\hline Eu me sinto feliz na maior parte do tempo. & & & $-0,64$ & & 0,39 \\
\hline Eu me irrito facilmente. & & & 0,30 & & 0,12 \\
\hline Eu raramente fico animado. & & & 0,59 & & 0,34 \\
\hline Bater em uma criança é a melhor maneira de ensiná-la & & & & 0,40 & 0,21 \\
\hline $\begin{array}{l}\text { O problema com as crianças, atualmente, é que seus pais } \\
\text { não as castigam o suficiente. }\end{array}$ & & & & 0,58 & 0,41 \\
\hline Meus pais eram sempre rígidos comigo. & & & & 0,30 & 0,96 \\
\hline Número de itens & 4 & 3 & 5 & 3 & \\
\hline Eigevalues (autovalores) & 2,62 & 2,25 & 1,58 & 1,53 & \\
\hline Alfa de Cronbach & 0,10 & $-0,35$ & $-0,04$ & 0,45 & \\
\hline Percentual de variância explicada & 10,07 & 8,68 & 6,10 & 5,91 & \\
\hline
\end{tabular}

\section{Discussão}

Hipóteses acerca dos resultados encontrados merecem destaque. Pela análise da escala sob o ponto de vista da literatura sobre construção de instrumentos (Pasquali, 2010), concluiu-se que o instrumento não atende a todos os critérios estabelecidos como fundamentais para a construção de medidas em psicologia, relacionadas a seguir. Critério de simplicidade, que enfatiza que cada item deve cobrir apenas um problema. Ao se analisar o instrumento deste estudo, verifica-se a ausência desse critério, como no item (Eu raramente me sinto ansioso ou com medo). Esse item apresenta duas ideias distintas, uma vez que a pessoa pode sentir-se ansiosa, mas não com medo, e viceversa. A existência de itens complexos pode levar o respondente à dúvida na escolha de uma resposta para ambas as situações. Critério da clarez̧a, que está relacionado a evitar itens negativos. Nota-se que no instrumento há itens negativos (Eu não me importaria se meu marido, ou esposa, tivesse amigos do sexo oposto; $\mathrm{Eu}$ não me preocupo muito; $\mathrm{Eu}$ não sinto muito prazer em conversar com as pessoas e As pessoas não devem se preocupar com o que os outros fazem). Por ser uma escala do tipo likert de 7 pontos, que vai de acordo total a desacordo total, concordar ou discordar com uma frase negativa pode dificultar a interpretação e pode induzir os respondentes ao erro. Critério da relevância, que sinaliza que o item deve ser consistente com o construto em questão, requisito representado pela carga fatorial do item em cada fator. Critério da modalidade, que ressalta a importância de se evitar o uso de expressões extremadas. Destaca-se, nesse instrumento, a ocorrência de itens com expressões extremadas (Eu não me preocupo muito; $\mathrm{Eu}$ raramente me sinto ansioso ou com medo; Eu frequentemente me preocupo com as coisas que podem dar errado e Eu raramente fico animado). Ao se considerar que a intensidade da resposta é dada na escala de resposta, e se o item apresenta-se de forma extremada, é de se esperar que haja dúvidas no momento da escolha da resposta que reproduza exatamente aquilo que se quer transmitir.

Além desses critérios, outro aspecto que merece atenção é a existência de itens excessivamente subjetivos; como exemplos têm-se os itens: "Estar em situações emocionais tensas me assusta", "Eu não me preocupo muito", "As pessoas não devem se preocupar com o que os outros fazem" e "Eu sou uma pessoa tradicional". Esses itens não são claros e objetivos e podem também depender do contexto cultural de cada respondente. A subjetividade dos itens pode ser considerada um problema do instrumento original que dificulta a adaptação para um contexto cultural diferente (Pasquali, 2010).

Vale ressaltar que, além desses aspectos, neste estudo para avaliação da fidedignidade da escala, a técnica estatística escolhida foi diferente da técnica sugerida pelos autores do instrumento. No estudo original os autores, apesar de utilizarem o cálculo do 
alfa de Cronbach, defendem que o cálculo do testereteste é a maneira mais adequada de se avaliar a confiabilidade da escala, pelo fato de que foram adotados procedimentos empíricos para a construção dos itens e também por a escala comportar mais de um fator, o que, do ponto de vista dos autores, favoreceria a técnica escolhida (Matsumoto \& LeRoux, 2006). Neste presente estudo, optou-se por utilizar somente o cálculo do alfa de Cronbach no estudo da confiabilidade do instrumento, por ser uma técnica recomendada pela maioria dos investigadores da área de psicometria e por ser uma técnica adequada aos propósitos desta pesquisa. Nos estudos divulgados pelos autores do instrumento (Matsumoto \& LeRoux, 2006), nas fases de construção e validação, não há dados que confirmem a existência de valores satisfatórios de alfas em amostras adequadas aos propósitos de validação de instrumentos de medida. Além disso, os autores não apresentam em nenhum estudo os valores de confiabilidade de cada fator, apenas o da escala toda.

Ademais, os resultados encontrados por este estudo não apresentaram valores de alfas satisfatórios, tendo sido encontrados valores de alfa negativos para dois fatores da escala. A ocorrência de alfas negativos, de acordo com Maroco e Garcia-Marques (2006), ocorre quando as correlações item-item são, de fato, negativas. A presença de alfas negativos pode ser corrigida por meio da recodificação com a inversão dos pontos como forma de garantir que os itens sejam posicionados na mesma direção. Conforme relatado, esse procedimento foi realizado e não se observou nenhuma mudança significativa que justificasse a recodificação dos pontos. De acordo com os mesmos autores (Maroco \& Garcia-Marques, 2006), alfas muito baixos ou negativos podem significar que os itens não medem o mesmo construto e é necessária uma reavaliação da base teórica que motivou a construção do instrumento.

Assim, o ponto que parece merecer maior destaque é a questão das bases teóricas que sustentam a construção do instrumento. Como relatado neste trabalho, o campo teórico sobre potencial de ajustamento intercultural e até mesmo o ajustamento em si permanecem obscuros e com diversas lacunas conceituais, especialmente no cenário brasileiro. Para Pasquali (2010), a ausência de uma teoria sólida sobre um construto parece ainda ser um dos grandes problemas dos instrumentos psicológicos. Uma teoria consistente sobre determinado traço psicológico permite ao pesquisador realizar uma definição constitutiva (situa o construto dentro da teoria) adequada aos construtos estudados e facilita a operacionalização dos itens, procedimentos estes essenciais para a construção de instrumentos de medida (Pasquali, 2010). Assim, argumenta-se como provável razão para a ocorrência das limitações e dos resultados insatisfatórios encontrados neste estudo a possível fragilidade teórica tanto na construção do instrumento - admitida pelos autores que buscaram fontes diversas para a elaboração dos itens - quanto na adequação do tema à realidade brasileira, provavelmente menos afeita a adaptações transculturais que os indivíduos estadunidenses, alvos primários da medida.

\section{Considerações finais}

Acredita-se que são necessários novos estudos que possam contribuir para se compreender melhor a base teórica sobre a temática abordada aqui. Da mesma maneira, uma exploração ampla e profunda das bases conceituais da temática se faz imprescindível para que haja uma melhor adequação dos itens que compõem a escala para alcançar uma representação mais fiel às necessidades psicológicas consideradas no campo teórico.

Sugere-se que o instrumento seja revisado teórica e empiricamente, já que, de acordo com a American Educational Research Association, American Association e a National Council on Measurement in Education (1999), as revisões são necessárias a partir do momento em que surgem novos dados de pesquisas sobre o instrumento, mudanças significativas no domínio ou novas condições para o uso e interpretação do teste. Essa revisão poderá resultar no refinamento deste instrumento ou construção de outros instrumentos que possam medir precisamente o construto pesquisado.

Vale salientar que este estudo contribuiu para divulgar os métodos utilizados para a adaptação cultural de instrumentos de medida elaborados em outros contextos. Apesar do avanço dos procedimentos estatísticos e da repercussão do processo de validação entre pesquisadores das ciências humanas, os procedimentos formais, tal como foi descrito neste estudo, são muitas vezes ignorados ou utilizados de maneira inadequada pelos pesquisadores. A construção de instrumentos de forma intuitiva e a simples tradução de instrumentos elaborados em outros contextos é a prova de que ainda é preciso propalar o uso de medidas psicométricas para a utilização e construção de instrumentos psicológicos.

Contudo, este trabalho se faz bastante pertinente para o estudo sobre ajustamento intercultural e para a área da avaliação psicológica, uma vez que apresenta estratégias adequadas para adaptação de instrumentos em psicologia. Mesmo tendo sido encontradas limitações, espera-se que este trabalho sirva de base 
para despertar o interesse de pesquisadores brasileiros com relação a esta área de estudo e a construção ou validação de instrumentos sobre o tema desta pesquisa, além da divulgação de procedimentos de adaptação cultural de instrumentos de medida.

\section{Referências}

Aaker, D. A., Kumar, V. \& Day, G. S. (2001). Marketing research. $7^{\mathrm{a}}$ ed. Nova Iorque: John Wiley and Sons.

American Educational Research Association, American Association \& National Council on Measurement in Education. (1999). Standards for educational and psychological testing. Nova Iorque: American Educational Research Association.

Beaton, D. E., Bombardier, C., Guillemin, F. \& Ferraz, M. B. (2000). Guidelines for the process of crosscultural adaptation of self-report measures. Spine, 25, 3186-3191.

Black, J. S. (1990). The relationship of personal characteristics with the adjustment of Japanese expatriate managers. Management International Review, 30(2), 119-134.

Brislin, R. (1981). Cross cultural encounters. Nova Iorque: Pergamon Press.

Hair, Jr., Black, W. C., Babin, B. J., Anderson, R. E. \& Tatham, R. L. (2006). Multivariate data analysis $\left(6^{a}\right.$ ed.). Upper Saddle River, NJ: Pearson Prentice Hall.

Maroco, J. \& Garcia-Marques, T. (2006). Qual a fiabilidade do alfa de Cronbach? Questões antigas e soluções modernas? Laboratório de Psicologia, 4(1), 65-90.

Matsumoto, D. \& LeRoux, J. A. (2006). Intercultural Adaptation Potential Scale. (Manual). Estados Unidos.

Matsumoto, D., Hirayama, S. \& LeRoux, J. A. (2005). Psychological skills related to intercultural adjustment. Em P. T. P. Wong \& L. C. J. Wong. (Eds.), Handbook of multicultural perspectives on stress and coping. Nova Iorque: Kluwer Academic/Plenum Publishing.

Matsumoto, D., LeRoux, J. A., Iwamoto, M., Choi, J. W., Rogers, D., Tatani, H. \& Uchida, H. (2003). The robustness of the Intercultural Adjustment Potential Scale (ICAPS): the search for a universal psychological engine of adjustment. International Journal of Intercultural Relations, 27, 543-562.
Matsumoto, D., LeRoux, J. A., Robles, Y., \& Campos, G. (2007) The Intercultural Adjustment Potential Scale (ICAPS) predicts adjustment above and beyond personality and general intelligence. International Journal of Intercultural Relations, 31(6), 747-759.

Matsumoto, D., LeRoux, J., Ratzlaff, C., Tatani, H., Uchida, H., Kim, C., \& Araki, S. (2001). Development and validation of a measure of intercultural adjustment potential in Japanese sojourners: the Intercultural Adjustment Potential Scale (ICAPS). International Journal of Intercultural Relations, 25, 483-510.

Nicholson, N. (1984). A theory of work role transitions. Administrative Science Quarterly, 29(2), 172-191.

Oberg, K. (1960). Culture shock: adjustment to new cultural environments. Practical Anthropology, 7, 177182.

Pasquali, L. (2005) Análise fatorial para pesquisadores. Brasília: LabPAM.

Pasquali, L. (2010). Instrumentação psicológica: fundamentos e práticas. Porto Alegre: Artmed.

Ruben, B. D. (1989). The study of cross-cultural competence: traditions and contemporary issues. International Journal of Intercultural Relations, 13, 229240.

Savick, V., Downing-Burnette, R., Hellen, H., Binden, F. \& Suntingen, W. (2004). Contrasts, changes, and correlates in actual and potential intercultural adjustment. International Journal of Intercultural Relations, 28, 311-329.

Shupe, E. I. (2007). Clashing cultures: a model of international student conflict. Joumal of CrossCultural Psychology, 38, 750-771.

Sussman, N. M. (2000). The dynamic nature of cultural identity throughout cultural transitions: why home is not so sweet. Personality and Social Psychology Review, 4(4), 355-373.

Tabachnick, B. \& Fidell, L. (2007). Using multivariate statistics. $5^{\mathrm{a}}$ ed. Boston: Allyn and Bacon.

Tomkins, S. S. (1963). Affect, imagery, and consciousness (Vol. 2: The negative affects). Nova Iorque: Springer.

Tsang, E. W. K. (2001). Adjustment of mainland Chinese academics and students to Singapore. International Journal of Intercultural Relations, 25, 347372. 
Ward, C. \& Searle, W. (1991). The impact of value discrepancies and cultural identity on psychological and sociocultural adjustment of sojourners. International Journal of International Relations, 15, 209-225.

Ward, C. (1996). Acculturation. Em D. Landis \& R. Bhagat (Eds.), Handbook of intercultural training (2a ed.). Newbury Park, CA: Sage.

Ward. C. \& Kennedy. A. (1993). Where's the "culture" in cross-cultural transition? Comparative studies of sojourner adjustment. Journal of Cross-Cultural Psychology, 24, 221-249.

Yoo, S. H., Matsumoto, D. \& LeRoux, J. (2006). Emotion regulation, emotion recognition and intercultural adjustment. International Journal of Intercultural Relations, 30(3), 345-363.

Zimmerman, S. (1995). Perceptions of intercultural communication competence and international student adaptation to an American campus.

Recebido em 28/11/2011

Communication Education, 44, 321-335.

Revisado em $12 / 12 / 2012$

Aprovado em 02/04/2013

Sobre os autores:

Jesselyn Nayara Tashima possui graduação e mestrado em Psicologia Social e do Trabalho pela Universidade Federal de Uberlândia.

Sinésio Gomide Junior possui graduação em Formação de Psicólogo, Mestrado e Doutorado pela Universidade de Brasília. Atualmente é professor associado da Universidade Federal de Uberlândia. Tem experiência na área de Psicologia, com ênfase em Psicologia do Trabalho e Organizacional, atuando principalmente nos seguintes temas: comportamento organizacional, psicometria, bem-estar no trabalho e gestão de recursos humanos.

\section{Contato com os autores:}

Av. Floriano Peixoto, 5.322 - Alto Umuarama

CEP 38405-184 - Uberlândia/MG

E-mail: jesse_tashima@yahoo.com.br 
